\title{
Transformação do significado de palavras sem sentido via relações arbitrárias de comparação com faces alegres
}

\author{
Transformation of the meaning of nonsense words through arbitrary \\ comparative relations with happy faces \\ Transformación del significado de palabras sin sentido vía relaciones \\ arbitrarias de comparación con rostros alegres
}

Gabriela Alves Rodrigues dos Santos ${ }^{1}$, William Ferreira Perez² ${ }^{2}$ João Henrique de Almeida ${ }^{3}$, Júlio C. de Rose ${ }^{4}$

[1-2] Paradigma - Centro de Ciências e Tecnologia do Comportamento[3-4] Universidade Federal de São Carlos [2-3-4] Instituto Nacional de Ciência e Tecnologia sobre Comportamento, Cognição e Ensino (INCT-ECCE) I Título abreviado: Relações arbitrárias de comparação I Endereço para correspondência: R. Vanderlei, 611 - Perdizes, São Paulo - SP, 05011-001 I Email: will.f.perez@gmail.com I DOI 10.18761/PAC.2017.028

Resumo: O presente estudo teve como objetivo investigar a transformação de significado de palavras sem sentido via relações de comparação estabelecidas com faces alegres. Adultos foram expostos a um treino em que duas figuras abstratas tinham função de dicas contextuais de comparação, controlando diferencialmente a escolha de diferentes quantidades de objetos. Em seguida, tais figuras foram utilizadas para estabelecer a seguinte rede relacional de comparação envolvendo palavras sem sentido e faces alegres: $\mathrm{A}<\mathrm{B}<\mathrm{FACE}$ ALEGRE $<\mathrm{D}<\mathrm{E}$. Depois de estabelecidas as relações arbitrárias de comparação e testadas as relações derivadas (e.g., A < E, E > A), os participantes avaliaram o significado dos estímulos A e E por meio do (a) diferencial semântico, de um (b) teste de preferência e do (c) IRAP. Os resultados do diferencial semântico demonstraram transformação de função dos estímulos A e E em acordo com relações de comparação. Neste instrumento, o estímulo E foi avaliado pelos participantes como mais positivo que o estímulo A, resultado confirmado estatisticamente quando comparado às avaliações do grupo controle. Os testes de preferência, embora tenham variado entre participantes, apresentam uma tendência de evitação do estímulo A e de preferência pelo estímulo E. Diferentemente das medidas anteriores, não foi possível verificar uma diferença clara entre as avaliações dos estímulos A e E por meio do IRAP, sendo ambos avaliados como igualmente positivos em termos de medida implícita. Os resultados observados demonstram que apesar de A ser avaliado como menos alegre do que E, este não foi considerado triste, produzindo a inconsistência observada no IRAP.

Palavras-chave: teoria das molduras relacionais, relações de comparação, faces alegres, diferencial semântico, teste de preferência, IRAP.

Nota dos autores: O presente estudo é parte da monografia de especialização da primeira autora, realizada no curso de clínica analítico-comportamental do Paradigma - Centro de Ciências do Comportamento. Dados preliminares dessa pesquisa foram apresentados na Encontro da ABPMC em 2016. Esta pesquisa é parte do programa científico do Instituto Nacional de Ciência e Tecnologia sobre Comportamento, Cognição e Ensino, apoiado pela Fundação de Amparo à Pesquisa do Estado de São Paulo (FAPESP, Processo \# 2014/50909-8) e pelo Conselho Nacional de Desenvolvimento Científico e Tecnológico (CNPq, Processo \# 465686/2014-1). João H. de Almeida é bolsista de pós-doutorado da FAPESP, Processo \# 2014/01874-7. 
Abstract: The present study aimed to investigate the transformation of meaning via comparison relations among meaningless and faces expressing happiness. Adults were exposed to a task in which two abstract figures were established as contextual cues controlling the selection of different quantities of multiple objects. Then, such stimuli were used to establish the following relational network of comparison involving nonsense words and faces expressing joy: $\mathrm{A}<\mathrm{B}<\mathrm{HAPPY} F A C E<\mathrm{D}<\mathrm{E}$. After establishing arbitrary relations of comparison and testing derived relations (e.g., $\mathrm{A}<\mathrm{E}, \mathrm{E}>\mathrm{A}$ ), the participants evaluated the meaning of stimuli A and E using (a) a semantic differential instrument, (b) a preference test and (c) an implicit test (IRAP). The results of the semantic differential suggest the transformation of the function of the stimuli A and $\mathrm{E}$ accordingly the comparison relation. In this instrument, stimulus E, a word related arbitrarily as being "more" than happy faces, was evaluated by participants as more positive when compared to stimulus A (established as "less" than happy faces). Preference test, although varying among participants, show a tendency to avoid stimulus A and a preference for stimulus E. Unlike the previous measures, it was not possible to verify a clear difference between the evaluations of stimuli A and E by means of the IRAP, both being evaluated as equally positive in terms of this implicit measure. The observed results demonstrate that although $\mathrm{A}$ is less happy than $\mathrm{E}$, it was not considered sad, producing this inconsistency in IRAP results.

Keywords: relational frame theory, comparison relations, happy faces, semantic differential, preference test, IRAP

Resumen: El presente estudio tuvo como objetivo investigar la transformación del significado mediante relaciones de comparación de palabras sin sentido con rostros alegres. Adultos fueron expuestos a un entrenamiento en el que dos figuras abstractas tenían función de claves contextuales de comparación, controlando diferencialmente la elección de diferentes cantidades de objetos. A continuación, estas figuras se utilizaron para establecer la siguiente red de relaciones de comparación que implicaba palabras sin sentido y caras alegres: $\mathrm{A}<\mathrm{B}$ $<$ ROSTRO ALEGRE $<\mathrm{D}<\mathrm{E}$. Después de establecer las relaciones arbitrarias de comparación y probar las relaciones derivadas (ej. $\mathrm{A}<\mathrm{E}, \mathrm{E}>\mathrm{A}$ ), los participantes evaluaron el significado de los estímulos A y E por medio de un diferencial semántico, de un (b) test de preferencia y del (c) IRAP. Los resultados del diferencial semántico demostraron la transformación de la función de los estímulos A y E de acuerdo con las relaciones de comparación. En este instrumento, el estímulo $\mathrm{E}$ fue evaluado por los participantes como más positivo que el estímulo A, resultado confirmado estadísticamente cuando se comparó con las evaluaciones del grupo control. Las pruebas de preferencia, aunque variaron entre participantes, presentan una tendencia de evitación al estímulo A y preferencia por el estímulo E. A diferencia de las medidas anteriores, no fue posible verificar una diferencia clara entre las evaluaciones de los estímulos A y E por medio del IRAP, siendo ambos evaluados como igualmente positivos en términos de medida implícita. Los resultados observados demuestran que a pesar de A ser menos alegre que E, este no fue considerado triste, produciendo esta inconsistencia en el IRAP.

Palabras-clave: teoría de los marcos relacionales, relaciones de comparación, rostros alegres, diferencial semántico, test de preferencia, IRAP. 
A equivalência de estímulo tem sido considerada um modelo comportamental do significado e, portanto, da relação palavra-referente (de Rose \& Bortoloti, 2007). Os estudos de equivalência demonstram que, uma vez ensinado um conjunto inicial de relações arbitrárias entre estímulos, por exemplo $\mathrm{AB}$ e $\mathrm{BC}$, os participantes (em geral humanos verbalmente competentes) são capazes de responder a novas relações derivadas das inicialmente ensinadas, por exemplo a relações simétricas, BA e $\mathrm{CB}$; transitivas, $\mathrm{AC}$ e $\mathrm{CA}$; e reflexivas: $\mathrm{AA}, \mathrm{BB}$ e $\mathrm{CC}$. Ao responder a novas combinações de estímulos, coerentes com as relações inicialmente treinadas, atesta-se a substitutabilidade, ou equivalência, entre os estímulos que foram arbitrariamente relacionados entre si no contexto do experimento (Sidman, 1994; Sidman \& Tailby, 1982).

Estudos demonstram, ainda, que uma vez que estímulos fazem parte da mesma classe de equivalência, certas funções comportamentais de um estímulo da classe (aversiva, reforçadora, eliciadora) podem ser estendidas para os demais membros da mesma classe (de Rose, McIlvane, Dube, Galpin \& Stoddard, 1988; Dougher, Augustson, Markham, Greenway \& Wulfert, 1994; Perez, Fidalgo, Kovac \& Nico, 2015; Perez, Tomanari \& Vaidya, 2015), caracterizando um processo denominado transferência de função.

A fim de testar experimentalmente o modelo de equivalência como um modelo de significado, Bortoloti e de Rose (2007) combinaram os procedimentos tradicionalmente empregados nos estudos sobre equivalência de estímulos com um instrumento de medida de significado, o diferencial semântico (Osgood \& Suci, 1952). Os autores realizaram um estudo com adultos, que foram divididos em dois grupos, um grupo experimental e um grupo controle. Os participantes do grupo experimental foram submetidos a um procedimento que envolveu o estabelecimento de relações de equivalência entre estímulos abstratos e expressões faciais de emoção. Foram estabelecidas as seguintes classes de estímulos, via tarefa de emparelhamento com o modelo (em inglês, matching-to-sample ou MTS): Face Alegre-B1-C1-D1, Face RaivosaB2-C2-D2 e Face de Nojo-B3-C3-D3. Os participantes que tiveram resultados positivos nos testes de equivalência foram então instruídos a avaliar, por meio do diferencial semântico, o significado dos estímulos D1, D2 e D3. O diferencial semântico apresentava o estímulo a ser avaliado acima de um conjunto de escalas bipolares de sete pontos, nas quais suas extremidades continham um par de adjetivos antônimos como, por exemplo, alegre/triste, tenso/relaxado, bonito/feio e etc. $\mathrm{O}$ grupo controle, que não foi submetido ao treino de relações arbitrárias, avaliou o significado dos estímulos abstratos e também das expressões faciais de emoção. Era esperado que o grupo controle avaliasse as faces como tendo algum significado e as figuras abstratas como neutras. Com relação à avaliação do grupo experimental, era esperado que os estímulos abstratos adquirissem um significado similar ao das faces - dada a realização de um treino de equivalência entre as faces e os estímulos abstratos para esse grupo. Em acordo com a hipótese do estudo, os resultados demonstraram significativa correspondência entre a avaliação das faces pelo grupo controle e a avaliação das figuras abstratas pelo grupo experimental. Verificou-se também que o grupo controle avaliou as figuras abstratas como sendo neutras. Esses resultados sugerem que houve transferência de função, ou seja, a extensão do significado do referente (faces) para seu símbolo correspondente (figuras abstratas), em uma simulação experimental estruturada com base no modelo de equivalência de estímulos.

Estes dados foram replicados por outros estudos usando a mesma metodologia (Bortoloti \& de Rose, 2008, 2009, 2011; Bortoloti, Rodrigues, Cortez, Pimentel \& de Rose, 2013; Bortoloti, Pimentel \& de Rose, 2014). Os estudos posteriores demonstraram algumas especificidades da transferência de significado das faces, tais como: (1) um efeito potencializador na transferência de significado quando o procedimento de emparelhamento com o modelo é realizado com atraso entre a apresentação do estímulo modelo e dos estímulos comparação (Bortoloti \& de Rose, 2009, 2012; de Almeida \& de Rose, 2015); (2) diferenças no grau de transferência proporcionalmente à distância nodal - assim quanto maior a distância nodal, menor a transferência de significado e vice e versa (Bortoloti \& de Rose, 2009); e (3) overtraining como uma variável capaz de potencializar a transferência de significado (Bortoloti et al., 2013). 
Segundo a Teoria das Molduras Relacionais - Relational Frame Theory (RFT) (Hayes, BarnesHolmes \& Roche, 2001; para artigos didáticos em Português, veja de Rose \& Rabelo, 2012 e Perez, Nico, Kovac, Fidalgo \& Leonardi, 2013), a equivalência de estímulos seria apenas uma entre diversas possibilidades de relações arbitrárias entre estímulos. Segundo os autores, humanos verbalmente competentes são capazes de responder a outras relações como, por exemplo, diferença, comparação, oposição, causalidade, relações temporais, de hierarquia e dêiticas (de perspectiva). Segundo a RFT, o comportamento de estabelecer relações arbitrárias é um operante denominado Responder Relacional Arbitrariamente Aplicável (RRAA). Tal operante é definido pelas seguintes propriedades funcionais: implicação mútua, implicação combinatória e transformação de função. A implicação mútua se refere à bidirecionalidade das relações entre estímulos, assim, se A é maior que $\mathrm{B}$, então B é menor que A; relações mutuamente implicadas também podem ser combinadas; assim, na implicação combinatória, se A é maior que $\mathrm{B}$ e $\mathrm{B}$ é maior que $\mathrm{C}, \log$ o $\mathrm{C}$ é menor que $\mathrm{A}$ e $\mathrm{A}$ é maior que $\mathrm{C}$; a transformação de função, por fim, descreve o efeito no qual, as funções comportamentais dos estímulos arbitrariamente relacionados são afetadas pelo tipo de relação arbitraria implicada; supondo que $\mathrm{A}$ é maior que $\mathrm{B}$ e $\mathrm{B}$ é maior que $\mathrm{C}$, caso $\mathrm{C}$ seja estabelecido como um estímulo aversivo, A e B serão progressivamente mais aversivos do que $\mathrm{C}$ (e.g., Dougher, Hamilton, Fink \& Harrington, 2007; Whelan, Barnes-Holmes \& Dymond, 2006).

Ampliando os achados de Bortoloti e de Rose $(2007,2009)$ com relações de equivalência, Perez, de Almeida e de Rose (2015) investigaram transformação de significado em relações de similaridade e oposição. O estudo foi realizado com adultos, divididos em um grupo controle e um grupo experimental. O grupo experimental foi submetido, inicialmente, a um procedimento para estabelecimento de dicas contextuais (figuras abstratas) que ocasionassem responder por igualdade ou por oposição. Nessa tarefa, uma de duas figuras abstratas era apresentada como dica contextual (IGUAL ou OPOSTO), seguida da apresentação de um estímulo modelo e de dois estímulos comparação sabidamente relacionados por igualdade e oposição (e.g., verão/inverno, rico/pobre, dia/ noite). Assim, quando a dica era IGUAL, escolher uma foto de verão na presença de um modelo verão era considerado correto; na presença da dica contextual para OPOSTO, a resposta reforçada era a escolha do estímulo comparação inverno. Uma vez verificado que as dicas contextuais abstratas controlavam responder por igualdade ou oposição, essas dicas foram utilizadas para estabelecer relações arbitrárias entre estímulos; as relações treinadas foram: A1 (face alegre) igual a B1; B2 oposto a A1 (face alegre); B1 igual a C1; B1 oposto $a$ C2. Na sequência, foram testadas as relações derivadas $\mathrm{C} 1$ igual a $\mathrm{B} 1, \mathrm{C} 2$ oposto a $\mathrm{B} 1, \mathrm{C} 1$ oposto a $\mathrm{B} 2, \mathrm{~B} 2$ oposto a $\mathrm{C} 1, \mathrm{C} 2$ igual a $\mathrm{B} 2$, e $\mathrm{B} 2$ igual a C2. Uma vez atestada a ocorrência de relações derivadas, os participantes do grupo experimental avaliavam os estímulos B1, B2, C1 e C2 por meio do diferencial semântico; o grupo controle, não submetido aos treinos anteriores, avaliou os mesmos estímulos e as faces alegres. O grupo experimental foi submetido, ainda, a um último procedimento, o Implicit Relational Assessment Procedure (IRAP; Barnes-Holmes, Barnes-Holmes, Power, Hayden, Milne, \& Stewart, 2006). Esse instrumento mensura a latência para a emissão de respostas relacionais quando o participante está sob pressão para responder com rapidez e precisão. Nesta fase eram apresentados na tela $\mathrm{C} 1$ ou $\mathrm{C} 2$ juntamente com faces alegres ou tristes. Como opções de resposta, os participantes tinham as palavras "similar" e "oposto". Os participantes ora eram requisitados a responder de acordo com o treino (bloco consistente) e ora de modo contrário (bloco inconsistente); nos blocos consistentes eles responderiam de acordo com o treino realizado nas fases anteriores - por exemplo, relacionando $\mathrm{C} 1$ e face alegre à Similar; já nos blocos inconsistentes, eles deveriam responder o inverso do treino - relacionando $\mathrm{C} 1$ e face alegre à Oposto. Os resultados no diferencial semântico demonstraram significativa correspondência entre a avaliação das faces pelo grupo controle e a avaliação das figuras abstratas relacionadas como iguais às faces alegres pelo grupo experimental; indicaram também que os estímulos abstratos arbitrariamente relacionados como opostos às faces alegres foram avaliados como sendo tristes. Os dados do IRAP indicaram 
que os participantes responderam mais rapidamente nos blocos consistentes quando comparados aos blocos inconsistentes, corroborando os resultados encontrados no diferencial semântico.

O experimento de Perez, de Almeida et al. (2015) demonstra transformação de função (i.e., do significado) com relações de igualdade e oposição. Outras relações, por exemplo as de comparação, também devem ser consideradas em uma explicação comportamental do significado. Esse é o caso quando aprendemos significados por gradação (e.g., bom, ótimo, excelente; ruim, péssimo, terrível), amplificando ou reduzindo variadas funções de estímulo. Diferentes estudos investigaram relações de comparação (e.g. Amd \& Barnes-Holmes, 2014; Amd \& Roche, 2015; Dougher et al., 2007; Perez et al., 2017; Whelan et al., 2006). Whelan et al. (2006), por exemplo, investigaram transformação de função consequencial com relações de comparação "mais que" e "menos que". Figuras abstratas foram inicialmente estabelecidas como ocasiões (dicas contextuais) para respostas de comparação "mais que" e "menos que", com base em propriedades físicas (diferentes quantidades), por meio de uma tarefa na qual eram exibidas figuras abstratas como estímulos modelo e diferentes quantidades de um dado objeto como comparação (por exemplo, três e cinco bolinhas; dois e sete quadrados, etc.). Na presença do modelo correspondente a "mais", a escolha da quantidade maior era considerada correta; na presença do modelo "menos", a escolha da menor quantidade era considerada correta. Uma vez verificado que as dicas contextuais abstratas controlavam respostas de comparação "mais" e "menos" com novos conjuntos de estímulos, essas dicas foram utilizadas para estabelecer as seguintes relações arbitrárias de comparação: $\mathrm{A}<\mathrm{B}<\mathrm{C}<\mathrm{D}<\mathrm{E}<\mathrm{F}<\mathrm{G}$ (sendo cada estímulo uma palavra sem sentido). Em seguida, foram testadas as relações derivadas (e.g., $\mathrm{A}<\mathrm{C}, \mathrm{D}>\mathrm{B}, \mathrm{G}<\mathrm{F}$, etc). Depois de estabelecidas e testadas as relações de comparação, o estímulo " $D$ ” da rede relacional comparativa foi estabelecido como reforçador condicionado. Os demais estímulos foram, então, apresentados em um teste de preferência. Os resultados mostraram que os participantes selecionaram consistentemente o estímulo em posição mais alta no ranking, demonstrando uma transformação de função consequencial de acordo com as molduras relacionais de comparação.
Estudos envolvendo relações arbitrárias de comparação também utilizaram expressões faciais de emoção (Amd \& Barnes-Holmes, 2014; Amd \& Roche, 2015). Amd e Roche (2015) verificaram que estímulos relacionados arbitrariamente à faces alegres podem ser avaliados como sendo mais ou menos alegres do que as faces em decorrência do estabelecimento de relações arbitrárias de comparação. A transformação de função foi verificada por meio de uma escala de auto-relato (Alegre ___|_|_|_ Não alegre), um teste de associação implícita (Implicit Association Test, IAT) e medidas eletrofisiológicas.

Considerando que a linguagem enquanto comportamento depende de inúmeros tipos de relações arbitrárias (Hayes et al., 2001), o presente estudo se propôs a estender os achados de Perez, de Almeida et al. (2015) aplicando a metodologia desenvolvida por Bortoloti e de Rose (2009), ou seja, o uso do diferencial semântico, ao estudo da transformação de significado dos estímulos em acordo com relações de comparação. Com base no procedimento proposto por Whelan et al. (2006), teve-se como objetivo investigar a transformação de significado de faces alegres por meio de um treino de relações arbitrárias entre estímulos em uma rede relacional de comparação. Diferentemente de Amd e Roche (2015), a medida de auto relato utilizada envolveu 11 diferentes escalas bipolares e uma outra medida implícita, o IRAP, foi utilizada (tal como em Perez, de Almeida e de Rose., 2015; ver também Bortoloti \& de Rose, 2012). A terceira medida empregada envolveu um teste de preferência.

\section{Método}

\section{Participantes}

Foram recrutados para a pesquisa 40 participantes, com idade entre 19 e 50 anos. Antes de iniciar o experimento, foi solicitado aos participantes ler e assinar um Termo de Consentimento Livre e Esclarecido (TCLE), aprovado pelo comitê de ética em pesquisa com humanos (Plataforma Brasil, CAAE 15687413.4.0000.5504). Os participantes foram distribuídos em três diferentes grupos, o Grupo Experimental $(\mathrm{N}=16)$ realizou todos os procedimentos programados. O Grupo Controle 1 $(\mathrm{N}=10)$ realizou apenas o teste de preferência e o 
Grupo Controle $2(\mathrm{~N}=14)$ realizou somente a avaliação dos estímulos abstratos utilizados no treino.

\section{Local, equipamentos e estímulos}

As sessões experimentais foram realizadas em uma sala silenciosa, com um computador e uma cadeira. O software RelationalFraming 1.0 (Perez, 2014), utilizado nas primeiras fases do experimento, permitiu a apresentação dos estímulos, o registro das respostas dos participantes e a apresentação de consequências durante as tarefas de matching-to-sample. O software que permite a aplicação do Procedimento Relacional de Avaliação Implícita - IRAP (Barnes-Holmes et al., 2006), foi utilizado nas últimas fases do experimento, para registrar e calcular as diferenças nas latências de resposta em diferentes tipos de tentativas. Também foi utilizado o diferencial semântico, apresentado impresso em folhas brancas A4.

\section{Procedimento}

Os participantes do Grupo Experimental foram submetidos à seguinte sequência de fases experimentais: (1) treino e teste de estabelecimento de dicas contextuais, (2) treino e teste de relações arbitrárias de comparação, (3) avaliação de significado pelo diferencial semântico, (4) teste de preferência e (5) IRAP. Os participantes do Grupo Controle 1 foram expostos somente às fases (3) avaliação de significado pelo diferencial semântico e o Grupo Controle 2 à fase (4) teste de preferência.

Fase 1: Estabelecimento da dica contextual. $\mathrm{O}$ objetivo dessa fase foi estabelecer duas figuras abstratas (pretas em fundo branco) como dicas contextuais para evocar as respostas relacionais de escolher, entre dois estímulos de comparação disponíveis, aquele que tivesse MAIS ou MENOS em relação à quantidade de elementos que o compunham. Foi utilizada uma tarefa de matching-to-sample (MTS) com duas escolhas (ver Figura 1).

Cada tentativa começava com a apresentação de uma forma abstrata como estímulo modelo, na parte superior da tela. Decorrido um intervalo de $1 \mathrm{~s}$, eram apresentados dois estímulos de comparação. Nesta primeira fase, os estímulos de comparação foram diferentes quantidades de um objeto (por exemplo: duas bolinhas e cinco bolinhas, um quadrado e seis quadrados; ver Whelan et al., 2006). A posição dos estímulos de comparação (à esquerda ou à direita) foi randomizada ao longo das tentativas. Para escolher dentre os estímulos comparação apresentados, o participante deveria clicar com o cursor do mouse em uma das figuras. Escolhas consideradas corretas foram seguidas da apresentação da palavra "correto", no centro da tela, por 1,5 s e da apresentação de um som suave. Escolhas incorretas foram seguidas da apresentação da palavra "incorreto" no centro da tela, e da apresentação de um som dissonante. Todas as tentativas foram seguidas por um intervalo entre tentativas (ITI) de $1 \mathrm{~s}$.

A tarefa foi iniciada com a seguinte instrução:

"Uma figura aparecerá no topo da tela, seguida da apresentação de duas outras figuras na parte inferior. Para cada símbolo apresentado na parte superior, existe apenas uma opção correta na parte inferior. Para escolher uma das opções, utilize o cursor do mouse e clique sobre ela. Sempre que você escolher a opção correta, o computador tocará um som suave e apresentará a palavra "CORRETO"; se você escolher a opção errada, um som dissonante será apresentado junto da palavra "INCORRETO". Haverá um momento, mais adiante, em que o computador não dirá se sua escolha foi correta ou não. Nesses casos, responda com base no que aprendeu anteriormente. O computador seguirá registrando suas respostas e avaliando seus acertos. Seu objetivo: acertar sempre”.

Dada a apresentação da dica contextual MAIS, a escolha do conjunto de estímulos com maior quantidade era considerada correta e a escolha da menor quantidade era considerada incorreta; dada a apresentação da dica contextual MENOS, a contingência descrita acima era revertida.

$\mathrm{Na}$ fase de treino foram apresentados até 48 tipos de tentativas com seis objetos diferentes (bolinhas, losangos, triângulos, quadrados, estrelas e linhas horizontais), sendo usadas três quantidades diferentes para cada objeto. Após atingir o critério de 32 acertos consecutivos, o participante iniciava a fase de teste, que consistia na apresentação de 32 tentativas, sem feedback, envolvendo diferentes quantidades de 4 objetos diferentes (cubos, pentágonos, sóis e linhas curvas). Caso o participante não acertasse pelo menos 95\% das tentativas de teste, a fase de treino e teste eram repetidas na mesma sequência. 


\section{Fase 1. Estabelecimento das dicas contextuais}
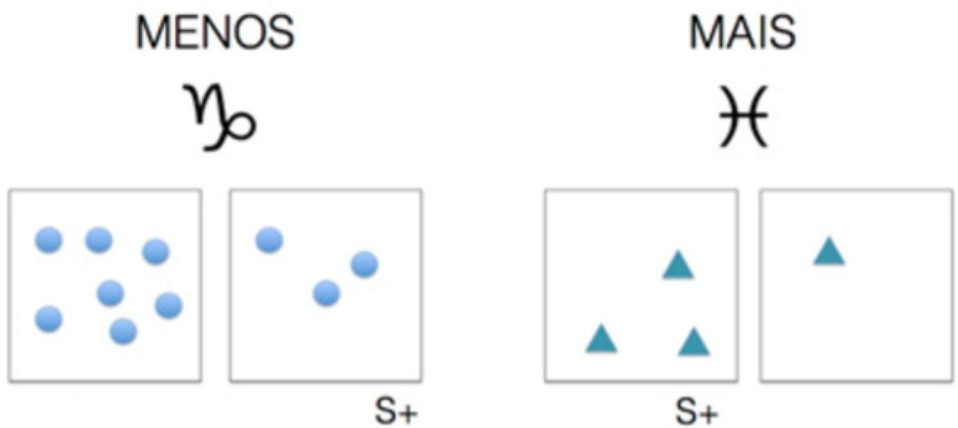

Fase 2. Treino e Teste das relaçöes arbitrárias

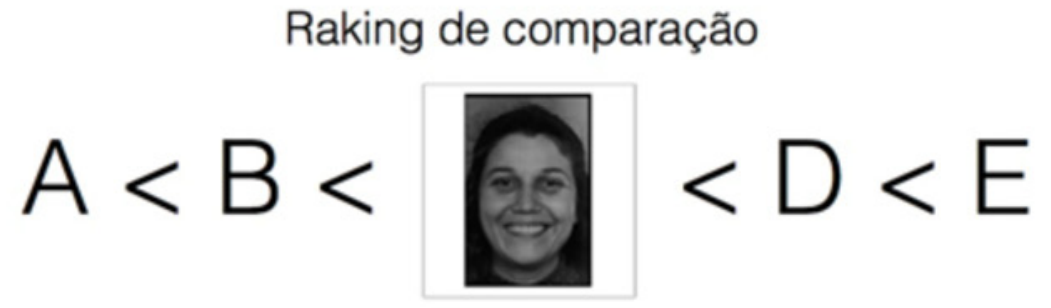

Tentativas de Treino (exemplos) Tentativas de Teste (exemplos)

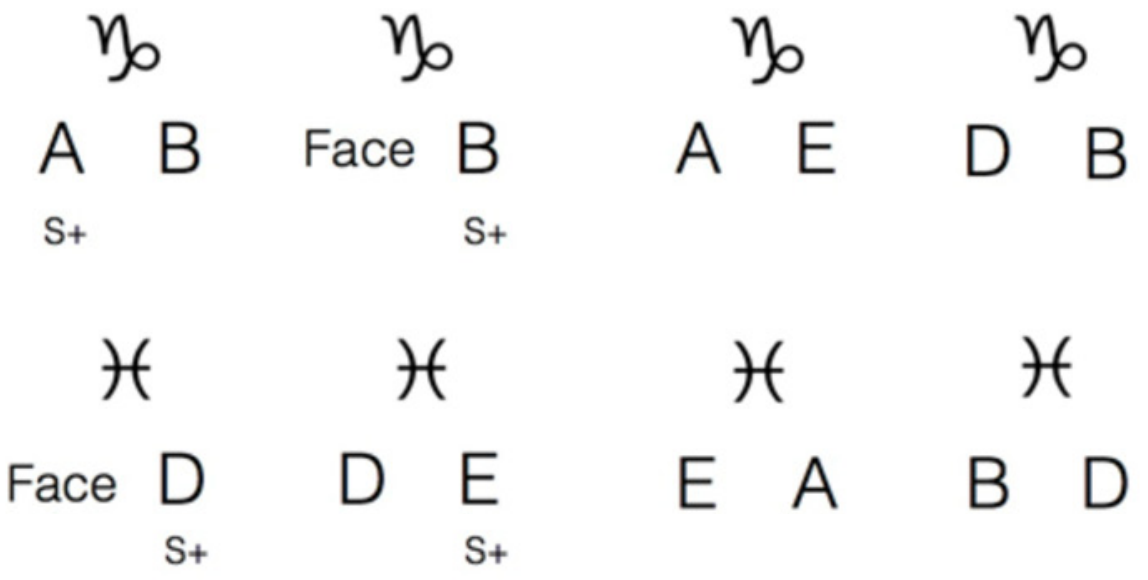

Figura 1. Exemplo de tentativas na Fase 1- Estabelecimento das dicas contextuais exemplos de tentativas na Fase 2 - Estabelecimento das relações arbitrárias de comparação

Fase 2: Estabelecimento das relações arbitrárias de comparação. Após atingir o critério na fase anterior, o treino de relações arbitrárias era iniciado sem nenhuma instrução. O objetivo desta fase era ensinar o participante a responder em conformidade com um ranking linear de uma rede relacional composta por quatro estímulos arbitrários (palavras sem sentido BTRECT, CIPHER, RIGUND, MURBEN) e faces expressando alegria (quatro faces extraídas a partir de Pictures of Facial Affect (C) CD-ROM, comprado do website de Paul Ekman, www.paulekman.com), como mostra a Figura 1. 
O ranking obedeceria a seguinte ordem: $\mathrm{A}<\mathrm{B}<$ Face $<\mathrm{D}<\mathrm{E}$. As palavras sem sentido foram aleatoriamente designadas como A, B, D ou E; as faces expressando alegria foram sempre designadas como C (o centro do ranking). Os parâmetros utilizados durante o treino e teste de relações arbitrárias foram os mesmos descritos para a fase anterior. Cada tentativa era iniciada com a apresentação de uma das dicas contextuais na parte superior da tela; na parte inferior, eram apresentadas duas das palavras sem sentido ou uma palavra sem sentido e uma das faces. Foram apresentadas quatro tipos de tentativas de treino representadas como [DICA(comparação 1, comparação2)], sendo o comparação correto sublinhado: [MENOS( $\underline{\text { A }}$, B)], [MENOS( $\underline{\mathrm{B}}, \mathrm{FACE})]$, [MAIS(FACE, $\underline{\mathrm{D}})]$ e [MAIS(D, E)] . Inicialmente, foram ensinadas as relações $\mathrm{B}$ menos que $\mathrm{FACE}$ e $\mathrm{D}$ mais que $\mathrm{FACE}$. As tentativas eram apresentadas randomicamente ao longo do treino e, depois que o participante acertasse 18 tentativas consecutivas, iniciava-se o treino das relações $\mathrm{A}$ menos que $\mathrm{B}$ e $\mathrm{E}$ mais que $\mathrm{D}$, até que o participante atingisse o mesmo critério. Em seguida, as quatro relações arbitrárias de comparação eram apresentadas conjuntamente, randomizadas, até que o participante atingisse o critério de 36 respostas corretas consecutivas.

Finalizado o treino relacional, os participantes eram imediatamente expostos à fase de teste; nessa fase, as respostas do participante não eram seguidas de consequências programadas para acerto e erro. Doze participantes (P1-P12) passaram por um teste resumido de derivação, no qual eram apresentadas as seguintes tentativas: [MENOS (A, E)] e [MAIS (E, A)]; cada tipo de tentativa era apresentada 10 vezes em ordem randomizada. Quatro participantes (P13-P16) passaram por um teste de derivação completo, com os seguintes tipos de tentativa: [MENOS (A, FACE)], [MENOS (FACE, E)], [MENOS (B, D)], [MENOS (A, E)], [MAIS (FACE, A)], [MAIS (E, FACE)], [MAIS (D, B)] e [MAIS (E, A)]. Cada tentativa era apresentada 8 vezes, totalizando 64 tentativas randomizadas. Encerrado o teste de derivação, os participantes que passaram pelo teste resumido seguiram diretamente para a próxima fase, independentemente do número de acertos no teste; no caso dos participantes que passaram pelo teste completo, as demais fases só foram realizadas com aqueles que acertaram pelo menos 60 em 64 tentativas.

\section{Fase 3: Avaliação dos estímulos A e E por meio} do diferencial semântico. Nesta etapa, cada participante era instruído a avaliar as palavras A e E de acordo com um conjunto de 11 escalas bipolares. Cada escala continha sete intervalos ancorados por adjetivos opostos: triste $\mathrm{x}$ alegre, tenso $\mathrm{x}$ relaxado, áspero $\mathrm{x}$ liso, lento $\mathrm{x}$ rápido, feio $\mathrm{x}$ bonito, pesado $\mathrm{x}$ leve, negativo $\mathrm{x}$ positivo, passivo $\mathrm{x}$ ativo, duro $\mathrm{x}$ macio, mau $\mathrm{x}$ bom e desagradável $\mathrm{x}$ agradável (Bortoloti \& de Rose, 2007; de Almeida, Bortoloti, Ferreira, Schelini \& de Rose, 2014). Metade dos participantes iniciou avaliando o estímulo A e a outra metade iniciou a avaliação pelo estímulo E. Cada folha de avaliação era entregue separadamente. Para instruções de aplicação e detalhes acerca do diferencial semântico, ver Bortoloti e de Rose (2009) e de Almeida et al. (2014).

Fase 4: Aplicação do teste de preferência para os estímulos A e E. Nesta fase do experimento, os participantes dos grupos experimental e controle eram requisitados a avaliar as palavras sem sentido de acordo com algum contexto. Cada participante recebia um conjunto de folhas com a seguinte instrução:

"Abaixo serão apresentadas algumas situações fictícias em que você precisará imaginar que algumas palavras se referem a lugares, pessoas, bebidas e marcas. Solicitaremos que você faça uma escolha entre elas. Não há respostas certas. O que queremos saber é a sua preferência. Pedimos que reflita sobre cada pergunta e procure responder com bastante sinceridade assinalando com um "X" a sua escolha".

Os testes de preferencia foram apresentados da seguinte forma: (1) "Imagine que BTRECT, CIPHER, RIGUND, MURBEN são lugares. Qual desses lugares você preferiria visitar? Qual desses lugares você não escolheria visitar?"; (2) "Imagine que BTRECT, CIPHER, RIGUND, MURBEN são pessoas. Qual delas você gostaria de se aproximar? Qual deles você teria menos vontade de se aproximar?"; (3) "Imagine que BTRECT, CIPHER, RIGUND, MURBEN são bebidas. Qual delas você preferiria provar? Qual delas você não faria questão de provar?"; (4)" Imagine que BTRECT, CIPHER, 
RIGUND, MURBEN são marcas de um produto. Qual deles você preferiria comprar? Qual você pensa que seria o pior produto dentre esses?".

\section{Fase 5: Avaliação dos estímulos A e E pelo Implicit} Relational Assessment Procedure (IRAP). Antes de iniciar o IRAP, foi dada aos participantes a seguinte instrução, conforme Perez et al. (2015):

Esta tarefa compreende blocos consistentes e inconsistentes de ensaios. Durante os blocos consistentes, respostas corretas irão corresponder às relações que você aprendeu na fase anterior do experimento; durante os blocos inconsistentes, você terá que responder invertendo as relações que você aprendeu. Blocos consistentes e inconsistentes serão sempre alternados, então, quando você terminar um dado bloco de tentativas, você vai saber o que esperar em seguida. Sempre que você responder de forma diferente do que a tarefa requer, um " $X$ " vermelho aparecerá e você precisará emitir a resposta correta tão rapidamente quanto possível. Esta tarefa também tem outra exigência: além de responder de forma precisa, você deve responder rapidamente. Se você demorar muito tempo para dar uma resposta, o computador mostrará a mensagem "muito lento"; isso significa que você precisa responder de forma mais rápida nas próximas tentativas. Uma vez que é uma tarefa comple$\mathrm{xa}$, teremos um pouco de prática no início. Durante a prática, eu estarei com você para tirar possíveis dúvidas e, uma vez que você aprender como realizar a tarefa, eu vou deixá-lo sozinho. (p. 11)

Os participantes foram expostos a blocos de 32 tentativas, que poderiam ser consistentes ou inconsistentes com as relações que foram treinadas. Como mostra a Figura 2, a cada tentativa, uma face alegre ou neutra (dos mesmos quatro atores utilizados no treino) era apresentada na parte superior da tela; no centro era apresentada uma das palavras sem sentido, A ou E; nos cantos inferiores esquerdo e direito eram apresentadas, randomicamente, as opções de resposta V (verdadeiro) ou F (falso). Foi requisitado que o participante escolhesse a opção "verdadeiro" ou "falso" para cada relação entre os estímulos A ou E e uma das faces (alegres ou neutras), de forma rápida e precisa. Respostas corretas eram seguidas da retirada dos estímulos e de um breve intervalo entre tentativas de $400 \mathrm{~ms}$ (IET). Respostas incorretas eram seguidas da apresentação de um X vermelho no centro da tela; os estímulos não eram retirados até que a resposta correta para aquela tentativa fosse emitida (e então seguida da retirada dos estímulos e do IET).

\section{Tentativas do IRAP}

Consistentes
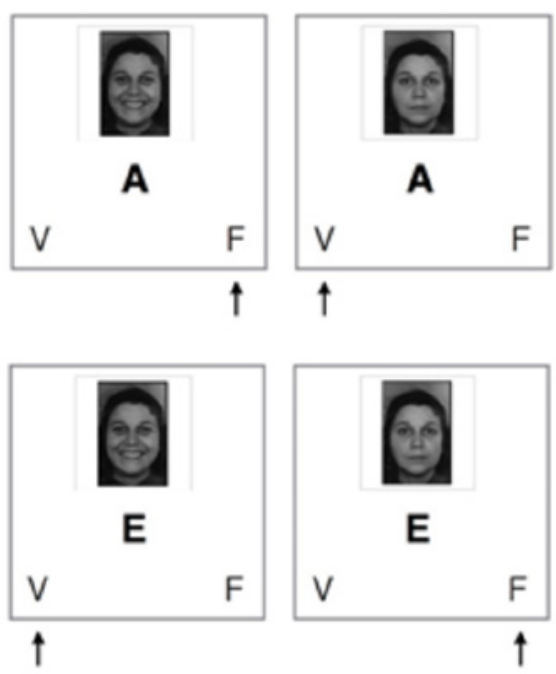

Inconsistentes
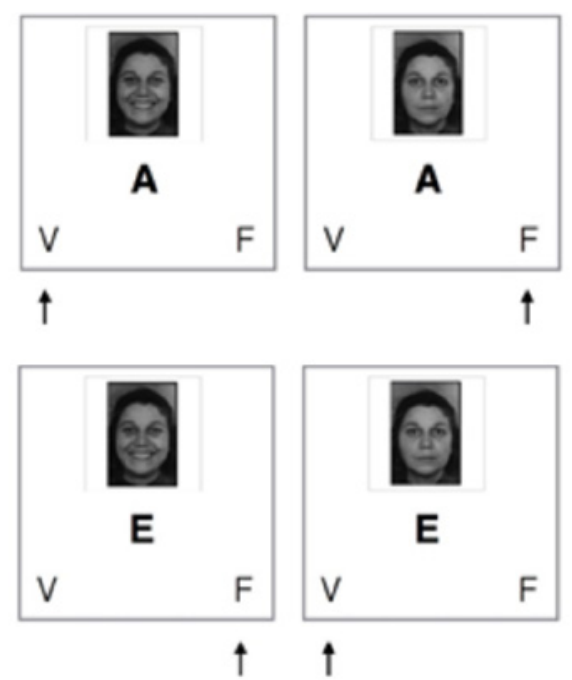

Figura 2. Tipos de tentativas consistentes e inconsistentes do IRAP. 
Como mostra a Figura 2, nos blocos consistentes, foram consideradas respostas corretas: Alegre-A/Falso, Neutra-A/Verdadeiro, Alegre-E/ Verdadeiro, Neutra-E/Falso; nas tentativas dos blocos inconsistentes, as contingências programas foram revertidas: Alegre-A/Verdadeiro, Neutra-A/ Falso, Alegre-E/Falso, Neutra-E/Verdadeiro. Blocos consistentes e inconsistentes sempre eram apresentados de maneira alternada. Metade dos participantes iniciou o IRAP pelos blocos consistentes e a outra metade pelos blocos inconsistentes.

O primeiro conjunto de blocos de tentativas tinha por objetivo ensinar o participante a responder na tarefa (fase de treino). O primeiro par de blocos consistente e inconsistente era apresentado sem nenhuma exigência de latência de resposta. Esse primeiro par tinha por objetivo ensinar ao participante a reversão das contingências de reforço após o término de cada bloco. Depois de apresentado o primeiro par de blocos de tentativas da fase de prática, a exigência de latência máxima de $2000 \mathrm{~ms}$ era inserida. Assim, caso o participante ultrapassasse esse intervalo para responder em $\mathrm{V}$ ou $\mathrm{F}$, um aviso seria apresentado no centro da tela "Tente responder mais rapidamente”. Uma vez iniciada a exigência de latência, o participante poderia ser submetido a no máximo 3 pares de blocos de tentativas consistente e inconsistente, até que atingisse o critério de $85 \%$ de respostas corretas com latência médias inferior a $2000 \mathrm{~ms}$ durante um par de blocos. Caso o participantes não atingisse esse critério, ele seria dispensado dos blocos de teste do IRAP. Os participantes que atingissem o critério, por sua vez, eram imediatamente (sem interrupção ou instrução) submetido a 3 pares de blocos consistentes e inconsistentes que foram utilizados como blocos de teste. Finalizados os três pares de teste, o programa era encerrado e o experimento finalizado.

\section{Resultados}

Como mostra a Tabela 1, durante a Fase 1, todos os participantes atingiram critério no treino de estabelecimento das dicas contextuais; foram necessárias entre 28 e 71 tentativas para atingir o critério de aprendizagem. Durante o teste, em que novos objetos em diferentes quantidades foram apresentados, todos os participantes atingiram critério para prosseguir para a próxima fase. Dois participantes (P7 e P9), no entanto, necessitaram de uma repetição do treino e do teste para atingir o critério estipulado.

A Tabela 1 apresenta também os resultados da Fase 2, em que relações arbitrárias de comparação foram estabelecidas e seguidas do teste de relações derivadas. Os participantes levaram entre 73 e 165 tentativas para atingir critério no treino. No teste de derivação, 11 participantes apresentaram mais de $80 \%$ de acerto, atingindo critério para prosseguir para as demais fases (P1, P2, P3, P4, P5, P7, P9, P10, P11, P12 e P13). Cinco participantes (P6, P8, P14, P15 e P16), no entanto, apresentaram desempenho inferior e tiveram sua participação interrompida.

Dez dos 11 participantes que passaram no teste de derivação, também finalizaram as demais fases experimentais (DS, Preferência e IRAP). A exceção foi o participante $P 7$, que não manteve critério durante a tarefa do IRAP; por essa razão, seu dado foi desconsiderado. Assim, as análises apresentadas a seguir consideram os dados de 10 participantes: P1, P2, P3, P4, P5, P9, P10, P11, P12 e P13. A Figura 3 apresenta os resultados do teste de transformação de significado, medida pelo diferencial semântico. Nota-se uma diferença na valência das avaliações para os estímulos A e E. O estímulo "menos" que as faces alegres, ou seja, $A$, tendeu a ser avaliado negativamente na maioria das escalas; em contrapartida, o estímulo E, arbitrariamente designado como sendo "mais" que as faces alegres, foi avaliado positivamente para a maioria das escalas. Com a realização de um teste de Mann-Whitney, foram observadas diferenças significativas entre as médias das atribuições realizadas pelos participantes do grupo experimental comparadas as atribuições dos mesmos estímulos realizadas pelos participantes do grupo controle (Aexp x Acon p<0,001 U=4.142,000; Eexp $\mathrm{x}$ Econ $\mathrm{p}=0,003 \mathrm{U}=4.408,500$ )

A Figura 4 apresenta o resultado dos testes de preferência. Embora as escolhas dos participantes tenham sido distribuídas para todos os estímulos, e não somente para $\mathrm{A}$ e E, nota-se uma tendência recorrente ao longo de todos os testes: o estímulo A tente a ser mais escolhido nas tentativas que envolvem evitação (e.g., Qual você pensa que seria o pior produto dentre esses?"), enquanto o estímulo E tende a ser mais escolhido nas tentativas de preferência 
(e.g., Qual [dentre esses produtos] você preferiria comprar?). Para uma análise estatística do teste comportamental, foram atribuídos valores de um a quatro para os estímulos sem sentido $(A=1, B=2$, $\mathrm{D}=3$ e $\mathrm{E}=4$ ). As questões foram divididas em dois tipos diferentes, as de evitação e as de preferência. As médias das atribuições de cada um destes tipos de questão realizadas pelos participantes do grupo experimental foram comparadas pelas atribuições de um grupo controle, com o mesmo número de participantes, que realizou apenas o preenchimento deste questionário. Por meio do teste de MannWhitney foi possível observar diferenças significativas ao se comparar tanto as questões de evitação $(\mathrm{p}<0.05 \mathrm{U}=590)$ como também as questões de preferência $(p<0,001 U=448)$ nas comparações entre o grupo experimental e o grupo controle.

Tabela 1. Número de tentativas e porcentagem de acertos para cada participante nas Fases 1 e 2

\begin{tabular}{|c|c|c|c|}
\hline Participante & $\begin{array}{l}\text { Fase } 1 . \\
\text { Número de tentativas de treino } \\
\text { (quantidades) até o critério }\end{array}$ & $\begin{array}{l}\text { Fase } 2 . \\
\text { Número de tentativas de treino } \\
\text { arbitrário }\end{array}$ & $\begin{array}{l}\text { Fase 2. Porcentagem de acer- } \\
\text { tos nos testes de derivação }\end{array}$ \\
\hline $\mathrm{P} 1$ & 36 & 85 & $16(80 \%)$ \\
\hline $\mathrm{P} 2$ & 36 & 73 & $20(100 \%)$ \\
\hline P3 & 36 & 97 & $16(80 \%)$ \\
\hline $\mathrm{P} 4$ & 36 & 89 & $20(100 \%)$ \\
\hline P5 & 36 & 85 & $20(100 \%)$ \\
\hline P6 & 36 & 99 & $14(70 \%)$ \\
\hline P7 & $57^{*}$ & 93 & $20(100 \%)$ \\
\hline P8 & 36 & 77 & $11(55 \%)$ \\
\hline P9 & $71^{*}$ & 85 & 19 (95\%) \\
\hline P10 & 36 & 75 & $20(100 \%)$ \\
\hline $\mathrm{P} 11$ & 28 & 84 & $18(90 \%)$ \\
\hline P12 & 36 & 93 & 19 (95\%) \\
\hline P13 & 28 & 86 & $61(95 \%)$ \\
\hline $\mathrm{P} 14$ & 28 & 156 & $26(40 \%)$ \\
\hline P15 & 28 & 89 & $39(60 \%)$ \\
\hline P16 & 28 & 165 & $26(40 \%)$ \\
\hline
\end{tabular}

Nota. O asterisco indica que o teste foi repetido. 

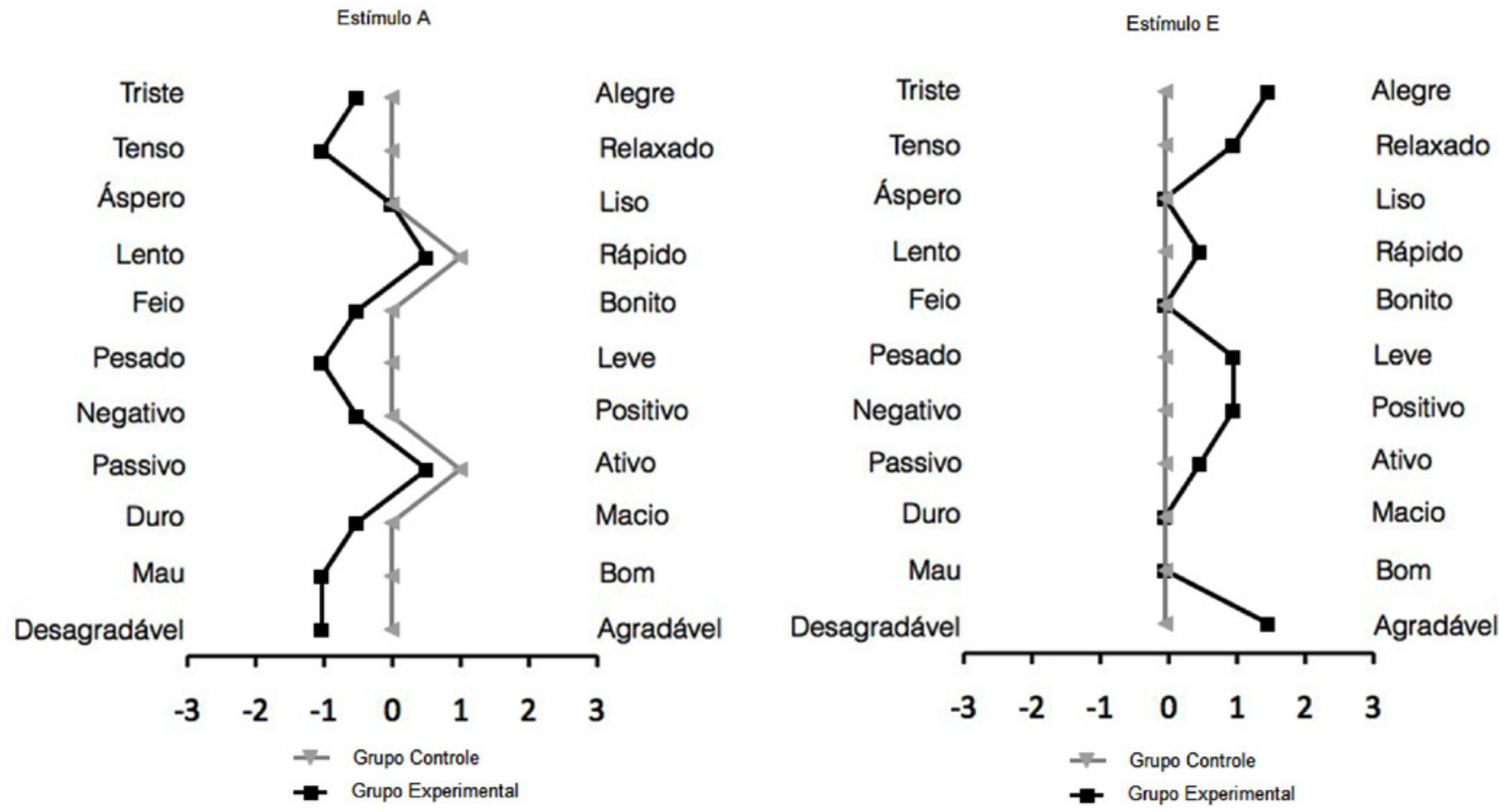

Figura 3. Mediana das avaliações dos estímulos A (linha preta) e E (linha cinza) ao longo das escalas bipolares do diferencial semântico.

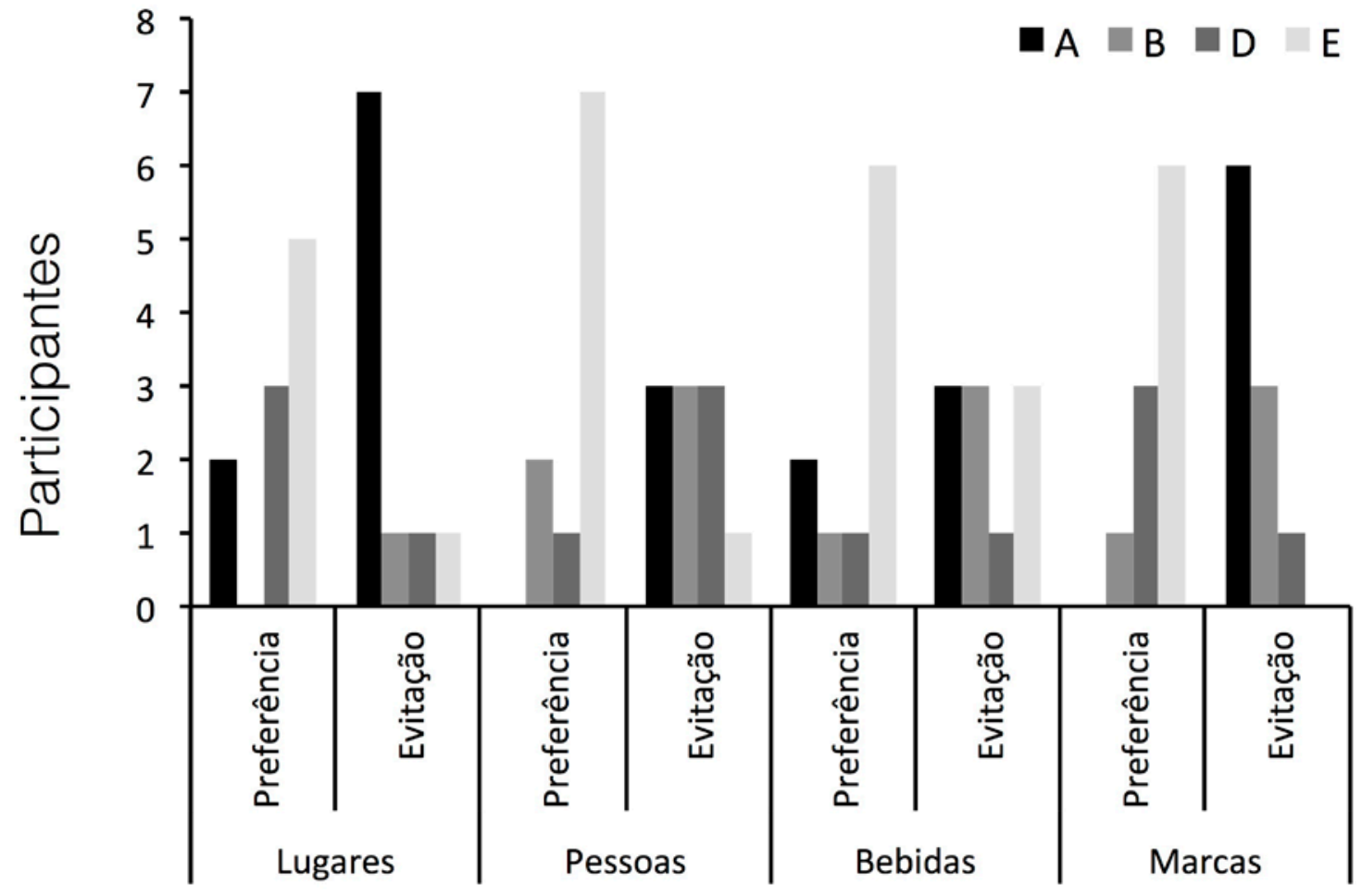

Figura 4. Escolhas dos participantes no teste de preferência pelos estímulos A, B, D e E. 
Os dados do IRAP são apresentados na Figura 5. Foram analisados os $\mathrm{D}_{\text {IRAP }}$ escores obtidos durante os blocos de teste. Escores médios próximos a zero sugerem que não há diferença na latência de resposta comparando os blocos consistentes e inconsistente. No entanto, na medida em que os escores médios se diferenciam de zero, é possível verificar latências menores para um dado tipo de bloco, no caso a seguir, para os blocos consistente. Os resultados do IRAP indicam que os estímulos A e E foram relacionados $(\mathrm{V})$ às faces alegres, sendo os escores $\mathrm{D}_{\text {IRAP }}$ das tentativas A-Alegre e E-Alegre significativamente diferentes de zero de acordo com um teste $t$ (A Alegre: $t(10)=4.136, p<0.002$; E Alegre: $t(10)$ $=2.823, p<0.01)$. Nesse sentido, os escores $\mathrm{D}_{\text {IRAP }}$ indicam que, nessas tentativas, a latência de resposta para $\mathrm{V}$ foi significativamente menor se comparada à latência de resposta para F. Um pós teste confirmou que o tamanho do efeito foi igualmente significativo e grande, quando observado o valor do eta parcial quadrado $F(3,36)=7,28, p=.001, \eta_{\text {Partial }}^{2}=.378$. No entanto, não foi observada uma diferença significativa entre os escores $D_{\text {IRAP }}$ desses dois tipos de tentativa (A Alegre vs. E Alegre: $t(10)=0.2615, p$ $<0.7$ ); assim, pode-se dizer, em termos de medida implícita, que os estímulos A e E foram avaliados como sendo igualmente alegres. Não foi observada uma relação entre os estímulos A, E e as faces neutras. Nesse caso, respostas em V ou F tiveram latências similares e o $D_{\text {IRAP }}$ score, ficou indiferenciado, não sendo significativamente diferente de zero (A Neutra: $t(10)=0.088, p<0.9$; E Neutra: $t(10)=$ $2.823, p<0.8)$.

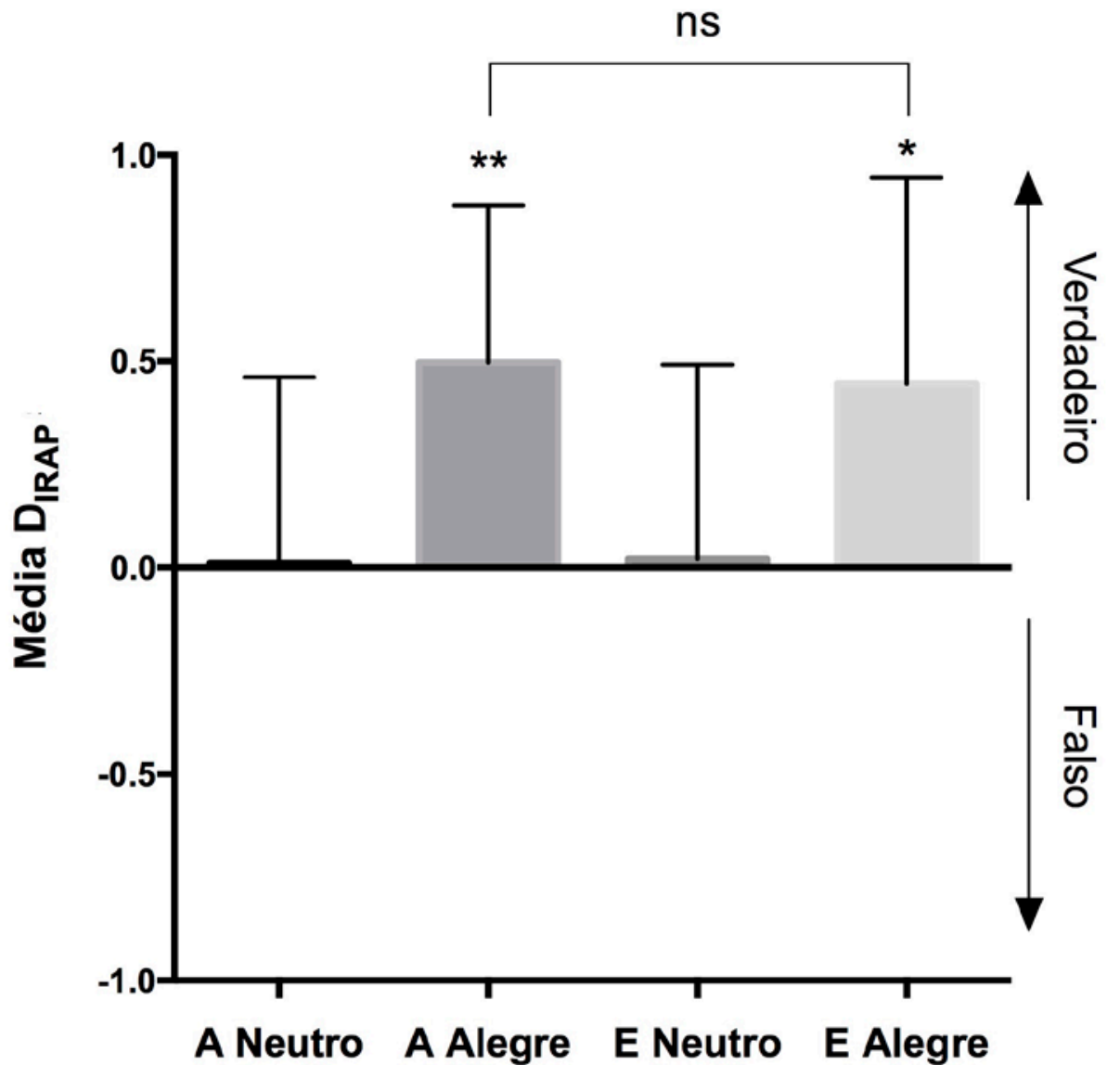

Tipos de tentativas do IRAP

Figura 5. Média dos D- ${ }_{\text {IRAP }}$ scores obtidos para os quatro tipos de tentativas do IRAP. D- ${ }_{\text {IRAP }}$ scores positivos, sinalizam respostas mais rápidas para "Verdadeiro" se comparado à latência média de respostas para "Falso". 


\section{Discussão}

O presente estudo teve como objetivo investigar a transformação de significado de faces alegres, por meio de um treino de relações arbitrárias entre estímulos, em uma rede relacional de comparação. Onze dos 16 participantes que passaram pelo treino relacional tiveram resultados positivos nos testes de derivação. Dez dos 11 participantes realizaram todas as etapas subsequentes envolvendo o DS, o teste de preferência e o IRAP. Os resultados do diferencial semântico sugerem que houve transformação de função dos estímulos A e E. Dessa forma, o estímulo E, uma palavra relacionada arbitrariamente como sendo "mais" do que as faces alegres, foi avaliado pelos participantes como sendo mais positivo (alegre, relaxado, bonito, bom e agradável) quando comparado ao estímulo A, uma palavra relacionada arbitrariamente como sendo "menos" do que as faces alegres. Na realidade, houve também uma transformação da valência dos estímulos, de tal forma que $\mathrm{A}$ foi avaliado como negativo e $\mathrm{E}$ como positivo. Os testes de preferência, embora tenham variado entre participantes, apresentam uma tendência de evitação para o estímulo A e preferência para o estímulo E. Diferentemente das medidas anteriores, não foi possível verificar uma diferença clara entre as avaliações dos estímulos A e E por meio do IRAP, sendo ambos avaliados como igualmente positivos em termos de medida implícita.

Os dados de treino e teste da Fase 1 corroboram estudos anteriores (e.g., Dymond \& Barnes, 1995; Dougher et al., 2007; Perez et al., 2017; Reilly et al., 2005; Whelan et al., 2006) que envolveram relações arbitrárias de comparação. Todos os participantes concluíram o treino e teste de estabelecimento das dicas contextuais e o treino de relações arbitrárias de comparação (e.g., Dymond \& Barnes, 1995; Dougher et al., 2007; Reilly et al., 2005; Perez et al., 2017; Whelan et al., 2006). Os dados dos testes de derivação, no entanto, foram negativos para alguns participantes, em especial aqueles que passaram pelo teste completo. Resultados negativos nos testes de derivação também foram encontrados em estudos anteriores (e.g., Whelan et al., 2006 - Experimento 1). Eventualmente, a repetição do treino e do teste pode ser necessária para obter o desempenho acurado (e.g., Whelan et al., 2006 -
Experimento 1). Perez et al. (2017) sugerem que uma diversificação do número de exemplares utilizados no estabelecimento da dica contextual pode afetar o desempenho no teste de derivação; em Perez et al. (2017), não somente diferentes quantidades foram empregadas, mas também conjuntos de estímulos de diferentes tamanhos, números e palavras envolvendo relações de comparação pré-experimentalmente estabelecidas. Estudos futuros poderiam replicar o presente procedimento avaliando se tal treino inicial afetaria o desempenho no diferencial semântico e, especialmente, no IRAP.

Os resultados deste estudo estendem os achados de Perez, de Almeida et al. (2015), pois demonstram a transformação de significado dos estímulos em acordo com um treino arbitrário de comparação, utilizando o DS como medida. Esse foi o primeiro estudo a aplicar a metodologia desenvolvida por Bortoloti e de Rose $(2007,2009)$ para a mensuração do significado dos estímulos pertencentes a redes relacionais de comparação. Os resultados obtidos no diferencial semântico replicam aqueles obtidos por meio de outras medidas, tais como escalas mais simplificadas, IAT e correlatos neurofisiológicos (Amd \& Barnes-Holmes, 2014; Amd \& Roche, 2015).

Assim como no diferencial semântico, a transformação de significado também foi verificada, embora menos claramente, em um teste de preferência. Foi possível observar uma maior preferência ou evitação em acordo com o treino arbitrário estabelecido, ou seja: preferência de E em relação a A e maior evitação de A se comparado a E. Um resultado que fortalece a interpretação dos dados de transformação de função é o da escolha do estímulo E nos testes de preferência ocorrendo com maior frequência do que a escolha dos estímulos que foram diretamente relacionados com as faces alegres (B e D). Esses dados também sugerem que as relações derivadas podem controlar respostas de escolhas em novos contextos, tal como tem sido relatado, por exemplo, na literatura sobre evaluative conditioning (Hughes, Barnes-Holmes, de Houwer, de Almeida \& Stewart, submetido).

Diferentemente de Perez, de Almeida et al. (2015), no entanto, não foram observadas diferenças no IRAP que sugerissem a transformação de função de A e E com base em relações de com- 
paração. Esse dado também difere daquele obtido com outro teste similar, o IAT (Amd \& BarnesHolmes, 2014; Amd \& Roche, 2015). As opções de resposta utilizadas no IRAP (Verdadeiro ou Falso) podem ter sido responsáveis por esses resultados, visto que não captam variações em um continuum da propriedade "alegre". Assim, mesmo que os estímulos $\mathrm{A}$ e $\mathrm{E}$ tenham sido relacionados respectivamente como "menos que" e "mais que" as faces alegres, respectivamente, as opções de resposta somente permitem capturar tal relação dicotomicamente: $\mathrm{V}$ ou $\mathrm{F}$, ou seja, foram relacionados ou não? Isso explicaria as respostas rápidas na opção "Verdadeiro", visto que ambos estímulos foram relacionados às faces expressando alegria. Outra interpretação possível para os dados do IRAP é a de que o treino relacional não foi suficiente para estabelecer as relações de comparação de tal modo que afetassem diferencialmente a probabilidade das respostas implícitas (como abordado no parágrafo anterior) - ou Respostas Relacionais Breves e Imediatas (BIRRs) (Hughes, Barnes-Holmes, \& Vahey, 2012). Um fato que pode ter contribuído com esse efeito é a duração do procedimento. No presente estudo o procedimento experimental foi realizado em uma única sessão, no experimento de Perez, de Almeida et al. (2015) as avaliações implícitas só foram executadas após duas sessões experimentais realizadas em dias diferentes. É possível que a reexposição realizada no experimento de similaridade e oposição tenha permitido o estabelecimento de uma história comportamental grande o suficiente para afetar as respostas relacionais breves e imediatas, o que não foi observado neste estudo.

Por fim, o presente estudo apresenta limitações que precisam ser superadas em estudos posteriores. O teste de derivação resumido, por exemplo, tal como utilizado para a maioria dos participantes, pode colocar em dúvida a natureza das respostas relacionais ensinadas. Tal argumento é fortalecido na medida em que somente um dos quatro participantes submetidos ao teste de derivação completo conseguiu atingir critério. Embora os dados de transformação de função do diferencial semântico assegurem a ocorrência de respostas relacionais de comparação, é necessário notar que o estímulo E, estabelecido como sendo mais alegre do que as próprias faces, atingiu um valor inferior ao frequentemente observado na avaliação desse tipo de faces em outros estudos (ver Bortoloti \& de Rose, 2009; Perez, de Almeida et al., 2015). Os resultados fracos nos testes de derivação também poderiam ser usados como explicação para a ausência de diferença nas avaliações implícitas de A e E durante o IRAP. Estudos futuros deverão replicar o presente procedimento assegurando que os participantes submetidos ao diferencial semântico tenham obtido critério em testes de derivação mais extensos, que incluam um número maior combinações das relações inicialmente treinadas.

\section{Referências}

Amd, M., \& Barnes-Holmes, D. (2014). A derived transformation of evaluative preferences using Implicit Association Tests. The Psychological Record,64(3), 475-485.

Amd, M., \& Roche, B.(2015). A derived transformation of valence functions across two 8-member comparative relational networks. The Psychological Record, 65.3 523-540.

Barnes-Holmes, D., Barnes-Holmes, Y., Power, P., Hayden, E., Milne, R., \& Stewart, I. (2006). Do you really know what you believe? Developing the implicit relational assessment procedure (IRAP) as a direct measure of implicit beliefs. The Irish Psychologist, 32, 169-177.

Bortoloti, R., \& de Rose, J. C. (2007). Medindo o grau de relacionamento de estímulos equivalentes. Psicologia: Reflexão e Crítica, 20, 252-258.

Bortoloti, R., \& de Rose, J. C. (2008). Transferência de "significado" de expressões faciais apresentadas brevemente para estímulos abstratos equivalentes a elas. Acta Comportamentalia, 16, 223-241.

Bortoloti, R., \& de Rose, J. C. (2009). Assessment of the relatedness of equivalent stimuli through a semantic differential. The Psychological Record, 59, 563-590.

Bortoloti, R., \& de Rose, J. C. (2011). Avaliação do efeito de dica semântica e da indução de significado entre estímulos abstratos equivalentes. Psicologia: Reflexão e Crítica, 24, 1-13.

Bortoloti, R., \& de Rose, J. C. (2012). Equivalent stimuli are more strongly related after training 
with delayed matching than after simultaneous matching: A study using the implicit relational assessment procedure (IRAP). Psychological Record, 62, 41-54.

Bortoloti, R., Rodrigues, N. C., Cortez, M. D., Pimentel, N., \& Rose, J. C. D. (2013). Overtraining increases the strength of equivalence relations. Psychology \& Neuroscience, 6, 357-364.

Bortoloti, R., Pimentel, N., \& Rose, J. C. D. (2014). Electrophysiological investigation of the functional overlap between semantic and equivalence relations. Psychology \& Neuroscience, 7, 183-191.

de Almeida, J. H., Bortoloti, R., dos Santos Ferreira, P. R., Schelini, P. W., \& de Rose, J. C. C. (2014). Análise da validade e precisão de instrumento de diferencial semântico. Psychology, 27(2), 272-281.

de Almeida, J. H., \& de Rose, J. C. (2015). Changing the meaningfulness of abstract stimuli by the reorganization of equivalence classes: effects of delayed mathcing. The Psychological Record.

de Rose, J. C. D., McIlvane, W. J., Dube, W. V., Galpin, V. C., \& Stoddard, L. T. (1988). Emergent simple discrimination established by indirect relation to differential consequences. Journal of the Experimental Analysis of Behavior, 50, 1-20.

de Rose, J. C. \& Bortoloti, R. (2007). A equivalência de estímulos como modelo do significado. Acta Comportamentalia, 15, 83-102.

de Rose, J. C., \& Rabelo, L. Z. (2012). Teoria das molduras relacionais e possíveis aplicações à educação. Revista de Deficiência Intelectual, 3, 10-15.

Dougher, M. J., Augustson, E., Markham, M. R., Greenway, D. E., \& Wulfert, E. (1994). The transfer of respondent eliciting and extinction functions through stimulus equivalence classes. Journal of the Experimental Analysis of Behavior, 62, 331-351.

Dougher, M. J., Hamilton, D. A., Fink, B. C., \& Harrington, J. (2007). Transformation of the discriminative and eliciting functions of generalized relational stimuli. Journal of the Experimental Analysis of Behavior, 88, 179-197.
Hayes, S. C., Barnes-Holmes, D., \& Roche, B. (2001). Relational frame theory: A post-Skinnerian account of human language and cognition. New York: Plenum.

Hughes, S., Barnes-Holmes, D., \& Vahey, N. (2012). Holding on to our functional roots when exploring new intellectual islands: A voyage through implicit cognition. The Journal of Contextual Behavioral Science, 1, 17-38.

Hughes, S., Barnes-Holmes, D., De Houwer, J., de Almeida, J. H. \& Stewart, I. (manuscrito submetido). On the symbolic generalization of likes and dislikes. Journal of Experimental Social Psycology.

Osgood, C. E., \& Suci, G. I. (1952). A measure of relation determined by both mean difference and profile information. Psychological Bulletin, 49, 251-262.

Perez, W. F. (2014). RelationalFraming (1.0) [computer software]. São Paulo, SP: Paradigma - Centro de Ciências do Comportamento.

Perez, W. F., de Almeida, J. H., \& de Rose, J. C. (2015). Transformation of meaning through relations of sameness and opposition. The Psychological Record, 65, 679-689.

Perez, W. F., Fidalgo, A. P., Kovac, R., \& Nico, Y. C. (2015). The transfer of Cfunc contextual control through equivalence relations. Journal of the Experimental Analysis of Behavior, 103, 511-523.

Perez, W. F., Kovac, R., Nico, Y. C., Caro, D. M., Fidalgo, A., Linares, I., de Almeida, J. H., \& de Rose, J. C. (2017). The transfer of Crel contextual control (same, opposite, less than, more than) through equivalence relations. Journal of the Experimental Analysis of Behavior, 108, 318334. doi: 10.1002/jeab.284

Perez, W. F., Nico, Y. C., Kovac, R., Fidalgo, A. P., \& Leonardi, J. L. (2013). Introdução à Teoria das Molduras Relacionais (Relational Frame Theory): principais conceitos, achados experimentais e possibilidades de aplicação. Perspectivas em Análise do Comportamento, 4, 33-51.

Sidman, M., \& Tailby, W. (1982). Conditional discrimination vs. matching-to-sample: An expansion of the testing paradigm. Journal of the Experimental Analysis of Behavior, 37, 261-273. 
Sidman, M. (1994). Equivalence relations and behavior: A research story. Boston: Authors Cooperative.

Whelan, R., Barnes-Holmes, D., \& Dymond, S. (2006). The transformation of consequential functions in accordance with the relational frames of more-than and less-than. Journal of the Experimental Analysis of Behavior, 86, 317-335.

Informações do Artigo

Histórico do artigo:

Submetido em: 05/12/2017

Primeira decisão editorial: 19/12/2017

Aceito em: 22/12/2017

Editor associado: Saulo Velasco 